Supplementary Information for:

\title{
Correlating calmodulin landscapes with chemical catalysis in neuronal nitric oxide synthase using time-resolved FRET and a 5-deazaflavin thermodynamic trap.
}

\author{
Tobias M. Hedison, Nicole G. H. Leferink, Sam Hay, Nigel Scrutton* \\ Manchester Synthetic Biology Research Centre for Fine and Speciality Chemicals (SYNBIOCHEM), Manchester \\ Institute of Biotechnology, Faculty of Life Sciences, University of Manchester, 131 Princess Street, Manchester \\ M1 7DN, United Kingdom, nigel.scrutton@manchester.ac.uk
}

Table of contents

1. Experimental Section.

1.1. Materials.

1.2. Cloning, expression, and purification of the C-terminal riboflavin kinase domain (tcRFK) from FAD synthetase isolated from C. ammoniagenes.

1.3. Expression and purification of native neuronal nitric oxide synthase ( $\mathrm{nNOS}$ ).

1.4. Cloning, expression, and purification of native and T34C/T110C calmodulin (CaM).

1.5. Biosynthesis of 5-deaza-FMN.

1.6. Circular dichroism (CD) measurements.

1.7. Steady-state activity measurements.

1.8. Static fluorescence measurements.

2. Results

2.1. Steady-state turnover.

2.2. Static fluorescence measurements.

2.3. Stopped-flow UV-Vis measurements.

2.4. Stopped-flow FRET measurements.

2.5. Circular dichroism measurements.

2.6. Absorbance measurements. 


\section{Experimental Section}

\subsection{Materials.}

All reagents were of analytical grade and were purchased from Sigma-Aldrich (Gillingham, Dorset, UK), unless otherwise stated. 5-deazariboflavin (5-dRF) was synthesized by Salford Ultrafine Chemicals and Research Limited (Manchester, UK). [4(R)-2H]NADPH (pro-R NADP2H) and [4(S) 2H] NADPH (pro-S NADP2H) were prepared and characterized as described previously. ${ }^{1}$ Alexa Fluor 555 C2 (A555) and Alexa 647 C2 (A647) maleimide were purchased from Thermo Fisher Scientific (Loughborough, UK). Pre-cast Sodium dodecyl sulphate polyacrylamide gel electrophoresis (SDS-PAGE) gels were purchased from Bio-Rad (Hempstead, UK).

\subsection{Cloning, expression, and purification of tcRFK.}

A synthetic gene encoding FAD synthetase from C. ammoniagenes ${ }^{2}$ was obtained from GenScript. A truncated version encoding the C-terminal riboflavin kinase domain (tcRFK) ${ }^{3}$ was amplified using the oligonucleotide primers tcRFK_fw (5'-GGGATTCATATGTTTTATGTTACCGGTCCG-3') and rFS-His_rv (5'CACACTCGAGTGATTCGGCTTGCAGAAAG-3'), and cloned between the Ndel and Xhol restriction sites of the pET22b(+) vector (Novagen, Prudhoe, UK) resulting in plasmid pET-tcRFK-His. Escherichia coli BL21(DE3) cells harbouring the pET-tcRFK-His plasmid were grown in 1 litre cultures of Luria-Bertani medium supplemented with $50 \mu \mathrm{g} \mathrm{mL} L^{-1}$ carbenicillin at $37^{\circ} \mathrm{C}$ until and $\mathrm{OD}_{600}$ of $\sim 0.6$ was reached. Protein expression was induced by the addition of $0.3 \mathrm{mM}$ isopropyl-d-1-thiogalactopyranoside (IPTG), and incubation was continued at $25^{\circ} \mathrm{C}$ for $16 \mathrm{~h}$ before harvesting the cells by centrifugation. The cell pellet was re-suspended in $50 \mathrm{mM} \mathrm{Tris-} \mathrm{HCl}(\mathrm{pH} 8.0)$ supplemented with $200 \mathrm{mM} \mathrm{NaCl}$ and $10 \mathrm{mM}$ imidazole, and the cells were broken by ultra-sonication. The lysate was cleared by centrifugation and loaded onto a Ni-IDA Agarose (Generon, Maidenhead, UK) column equilibrated with lysis buffer. The column was washed with $75 \mathrm{mM}$ imidazole in the same buffer, and the tcRFK protein was eluted with $250 \mathrm{mM}$ imidazole. The purified protein was dialysed against $50 \mathrm{mM} \mathrm{Tris-HCl}(\mathrm{pH} 8.0)$ supplemented with $200 \mathrm{mM} \mathrm{NaCl}$ and stored at $-20^{\circ} \mathrm{C}$. Protein concentrations were determined using a calculated molar extinction coefficient $\left(\varepsilon_{280}\right)$ of $14.44 \mathrm{mM}^{-1} \mathrm{~cm}^{-1}$ at $280 \mathrm{~nm}$.

\subsection{Expression and purification of native NOS.}

Native $\mathrm{His}_{6}$-tagged rat neuronal NOS was overexpressed in E. coli and purified by Ni-IDA Agarose affinity chromatography and 2',5'-ADP-Sepharose (GE Healthcare, Little Chalfont, UK) affinity chromatography as described previously. ${ }^{4,5}$ Protein concentrations were determined at $444 \mathrm{~nm}$ in the presence of carbon monoxide (CO), using a molar extinction coefficient of $74 \mathrm{mM}^{-1} \mathrm{~cm}^{-1}\left(A_{444}-A_{500}\right)$ for the ferrous heme-CO adduct. $^{6}$ Immediately prior to kinetic experiments, native NOS was incubated with $0.2 \mathrm{mM} F A D, 0.2 \mathrm{mM} F M N$, $0.2 \mathrm{mM}$ tetrahydobiopterin $\left(\mathrm{H}_{4} \mathrm{~B}\right)$ and $2 \mathrm{mM}$ DTT for $30 \mathrm{~min}$ on ice. Next, a few grains of potassium ferricyanide were added and excess reagents were removed by applying the protein solution onto a Econo-Pac DG10 gel filtration column (Bio-Rad, Hempstead, UK) equilibrated in $40 \mathrm{mM}$ HEPES (pH 7.6) buffer supplemented with $10 \%$ glycerol and $150 \mathrm{mM} \mathrm{NaCl}$. For anaerobic experiments, the final desalting step was performed in an anaerobic glove box (Belle Technology, Weymouth, UK) under nitrogen atmosphere in which oxygen levels were kept below 5 ppm. 


\subsection{Cloning, expression, and purification of native and T34C/T110C calmodulin (CaM).}

The CaM variant T34C/T110C was constructed using the wild-type CaM containing pCOLADuet-1 plasmid template by using the 'QuickChange' method (Agilent, Stockport, UK). Both recombinant mammalian CaM and the T34C/T110C were expressed in E. coli BL21(DE3) and purified using a single Phenyl-Sepharose hydrophobic interaction chromatography (GE Healthcare, Little Chalfont, UK) step as described before. ${ }^{4,7}$ Protein concentrations were determined at $276 \mathrm{~nm}$ using an extinction coefficient of $\varepsilon_{276}=3.006 \mathrm{mM}^{-1} \mathrm{~cm}^{-1}$.

\subsection{Biosynthesis of 5-deaza-FMN.}

For the biosynthesis of 5-deaza-FMN (5-dFMN), a solution containing $0.5 \mathrm{mM}$ 5-dRF, $1 \mathrm{mM} \mathrm{ATP,} 10 \mathrm{mM} \mathrm{MgCl}_{2}$ and $10 \mu \mathrm{M}$ tcRFK in $50 \mathrm{mM}$ Tris- $\mathrm{HCl}(\mathrm{pH} 8.0)$ was incubated at $37^{\circ} \mathrm{C}$ for 120 min while shaking. The reaction was stopped by incubating the solution for $5 \mathrm{~min}$ at $95^{\circ} \mathrm{C}$, and any insoluble material was removed by centrifugation. The reaction products were identified by thin-layer chromatography (TLC). Samples taken during the reaction, and the reference compounds FAD, FMN, riboflavin, and 5-dRF were applied onto a silica gel $60 F_{254}$ TLC plate (Merck Millipore, Nottingham, UK). Butanol:acetic acid:water (12:3:5) was used as mobile phase and fluorescent spots were visualized by UV illumination.

\subsection{Circular dichroism (CD) measurements.}

Circular dichroism was performed on an Applied Photophysics (Leatherhead, UK) Chirascan qCD spectrometer at $25{ }^{\circ} \mathrm{C}$ using a sealed cuvette with a $0.1 \mathrm{~mm}$ path length. Measurements were performed with $5 \mu \mathrm{M}$ native or 5-dFMN nNOS in 40 mM HEPES (pH 7.6), supplemented with $150 \mathrm{mM} \mathrm{NaCl}$ and $10 \%$ glycerol. Mean residual ellipicities (MRE, $[\theta]_{\mathrm{MR}}$ ) were calculated using eq 1 . Where $\theta$ is degrees of ellipicities, $I$ is the cuvette path length in $\mathrm{cm}$ and $C_{M R}$ is the mean residue concentration. $C_{M R}$ is calculated using eq. 2 where $n$ is the number of amino acids and $\mathrm{c}$ is the concentration of the protein in molar.

$[\theta]_{\mathrm{MR}}=100 \theta /\left(\mathrm{C}_{\mathrm{MR}} \mathrm{l}\right)$

$\mathrm{C}_{\mathrm{MR}}=\mathrm{nc} \quad($ eq S2)

\subsection{Static fluorescence measurements.}

Fluorescence emission spectra were recorded on an Edinburgh Instruments (Livingston, UK) FLS920 fluorometer equipped with double excitation and emission monochromators, a red-sensitive cooled photomultiplier detector, and a $450 \mathrm{~W}$ xenon arc lamp. Spectra were recorded using $0.5 \mathrm{~nm}$ excitation and $5 \mathrm{~nm}$ emission slit-widths in $1 \mathrm{~mL}$ fluorescent quartz cells (Starna Scientific Ltd, Hainault, UK) with a $10 \mathrm{~mm}$ excitation path length. Fluorescence emission data were collected at $25^{\circ} \mathrm{C}$ in $40 \mathrm{mM} \mathrm{HEPES}$ (pH 7.6), $150 \mathrm{mM}$ $\mathrm{NaCl}$ and $10 \%$ glycerol, unless otherwise stated. For $\mathrm{Ca}^{2+}$ free measurements a calcium sponge (Molecular Probes, Thermo Fisher Scientific - Loughbourough, UK) was used to remove the divalent ion from all buffers.

\subsection{Steady-state activity measurements.}


The steady-state turnover of native- and 5-dFMN reconstituted-nNOS was determined at $10{ }^{\circ} \mathrm{C}$ on assay mixtures containing catalytic amounts of NOS, $10 \mathrm{mM}$ L-arginine, $0.5 \mathrm{mM} \mathrm{CaCl}_{2}$, and $0.1 \mathrm{mM} \mathrm{NADPH}$, in the presence or absence of $7 \mu \mathrm{M} \mathrm{CaM}$ in assay buffer. Steady-state NADPH oxidation rates were determined at $340 \mathrm{~nm}\left(\varepsilon_{340}=6.22 \mathrm{mM}^{-1} \mathrm{~cm}^{-1}\right)$, cytochrome $c$ reduction at $550 \mathrm{~nm}\left(\Delta \varepsilon_{550}=21.1 \mathrm{mM}^{-1} \mathrm{~cm}^{-1}\right)$ in the presence of $10 \mu \mathrm{M}$ bovine heart cytochrome $c$, ferricyanide reduction at $420 \mathrm{~nm}\left(\varepsilon_{420}=1.04 \mathrm{mM}^{-1} \mathrm{~cm}^{-1}\right)$ in the presence of $1 \mathrm{mM}$ potassium ferricyanide, and NO formation was monitored at $401 \mathrm{~nm}\left(\Delta \varepsilon_{401}=38 \mathrm{mM}^{-1} \mathrm{~cm}^{-1}\right)$ in the presence of $10 \mu \mathrm{M}$ oxyhemoglobin. All steady state assays were conducted in $40 \mathrm{mM} \mathrm{HEPES}$ (pH 7.6) supplemented with $150 \mathrm{mM} \mathrm{NaCl}, 10 \%$ glycerol.

\section{Results}

\subsection{Steady State Turnover}

\subsection{1.}

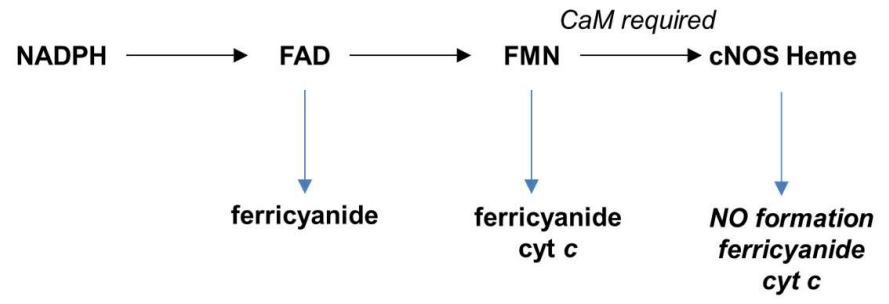

Scheme S1. Electron flow through the NOS di-flavin oxidoreductase to intrinsic and extrinsic electron accepting partners. Black and blue arrows are representative of electron flow through nNOS and from nNOS to redox partners, respectively. ${ }^{8} \mathrm{CaM}$ is required for cross-monomer electron transfer from nNOS FMN to heme.,10 CaM along with all electron accepting partners that require CaM-nNOS binding are shown in italics. 


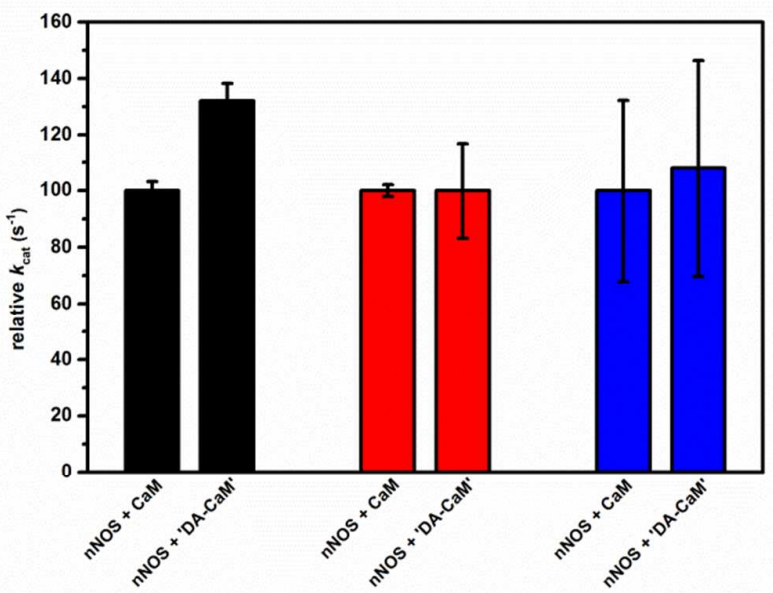

Figure S1. Comparison between native- and DA-T34C/T110C-CaM ('DA-CaM') bound nNOS steady-state turnover numbers. Black, red and blue bars represent NADPH consumption, NO formation and cyt $c$ reduction assays, respectively. Data are normalized to the native nNOS assay turnover numbers. 
Table S1. Steady state $k_{\text {cat }}$ values for nNOS turnover assays.

\begin{tabular}{lllll}
\hline NOS & $\begin{array}{l}\text { FeCN reduction } \\
\left(\mathrm{s}^{-1}\right)\end{array}$ & $\begin{array}{l}{ }^{*} \text { cyt } c \text { reduction } \\
\left(\mathrm{s}^{-1}\right)\end{array}$ & $\begin{array}{l}\text { NADPH } \\
\text { consumption }\left(\mathrm{s}^{-1}\right)\end{array}$ & NO formation $\left(\mathrm{s}^{-1}\right)$ \\
\hline Native & $32.8(1.1)$ & $0.4(0.03)$ & $0.05(0.00)$ & ND \\
Native + CaM & $32.5(0.7)$ & $1.6(0.6)$ & $0.27(0.01)$ & $0.09(0.002)$ \\
5-dFMN & $28.2(0.4)$ & ND & $0.003(0.000)$ & ND \\
5-dFMN nNOS + & $28.2(0.4)$ & ND & $0.003(0.000)$ & ND \\
CaM & & & &
\end{tabular}

All data are recorded at $10{ }^{\circ} \mathrm{C} .{ }^{*}$ cyt $c$ was shown to react with NADPH without nNOS present, so steady-state cyt $c$ reduction rates were calculated by subtracting rates of solution reaction from those with enzyme present. Estimated errors are given in parenthesis. ND, not detected. 
2.2 Static Fluorescence Measurements.

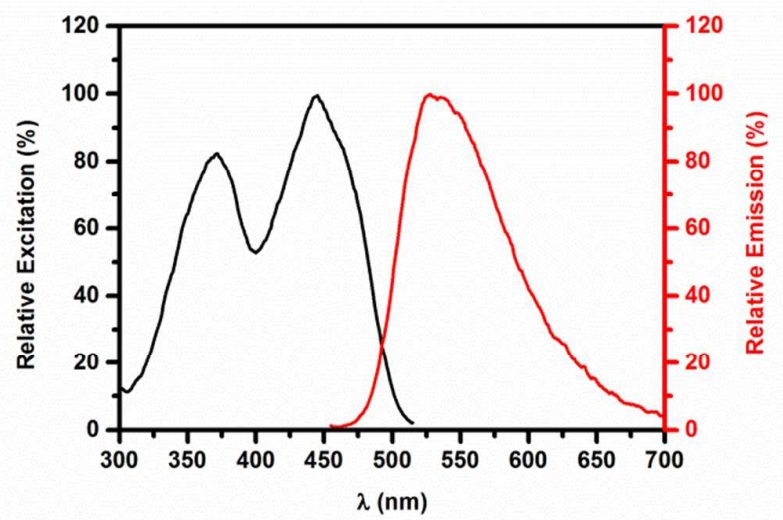

Figure S2. Fluorescence excitation and emission spectra of oxidized nNOS. Excitation wavelengths used in fluorescence experiments (555 and $645 \mathrm{~nm}$ ) to monitor nNOS-bound CaM dynamics are red shifted from intrinsic nNOS flavin chromophore excitation spectra (black). nNOS emission was recorded with a $450 \mathrm{~nm}$ excitation wavelength while excitation data was recorded with a $525 \mathrm{~nm}$ emission wavelength. All $1 \mu \mathrm{M}$ nNOS samples were in $40 \mathrm{mM}$ HEPES (pH 7.6) supplemented with $150 \mathrm{mM} \mathrm{NaCl}, 1 \mathrm{mM} \mathrm{CaCl}, 10 \%$ glycerol in the presence of $5 \mu \mathrm{M} \mathrm{H} \mathrm{H}_{4} \mathrm{~B}$. Data are normalized to $\lambda$-max values for both excitation and emission spectra. The appearance of nNOS-blue semiquinone (which has absorbance features between 550 and $650 \mathrm{~nm}$ ) is minimal during single-turnover stopped-flow measurements of nNOS flavin reduction thus there are no complications from direct excitation of nNOS semiquinone flavin species. ${ }^{11}$ 

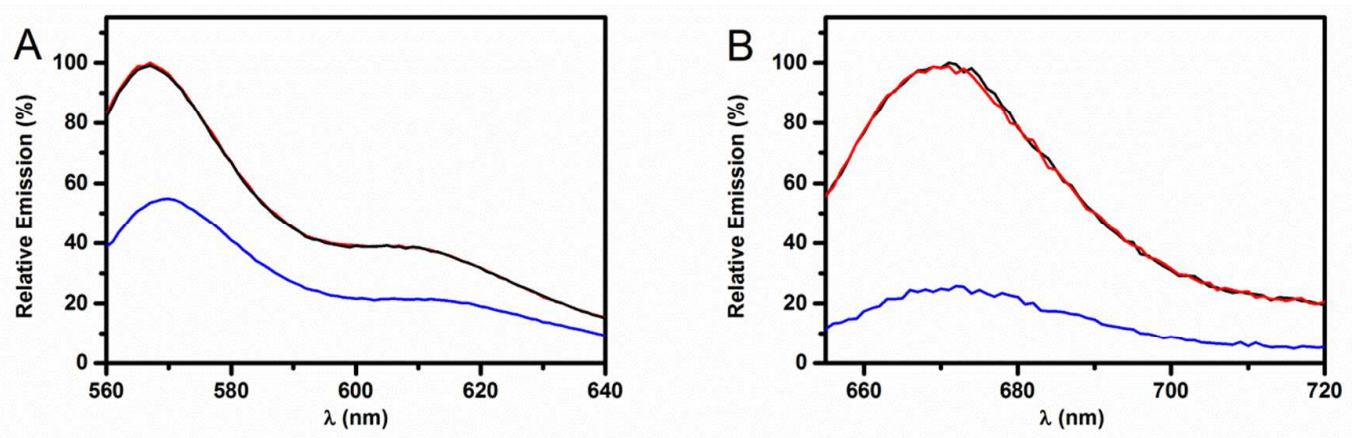

Figure S3. Fluorescence emission changes associated with the binding of A) $0.3 \mu \mathrm{M}$ D-T34C/T110C-CaM or B) $0.3 \mu \mathrm{M}$ A-T34C/T110C-CaM (black) with $\mathrm{Ca}^{2+}$ (red) or both $\mathrm{Ca}^{2+}$ and $0.3 \mu \mathrm{M}$ nNOS (blue). Data show both a quenching in the emission as well as a $\sim 3 \mathrm{~nm}$ red-shift in the $\lambda$-maxima of both donor and acceptor fluorophores when fluorophore labelled T34C/T110C-CaM binds to nNOS in the presence of Ca ${ }^{2+}$ relative to the $\mathrm{Ca}^{2+}$ free or $\mathrm{Ca}^{2+}$ bound fluorophore labelled T34C/T110-CaM. D-T34C/T110C- and A-T34C/T110C-CaM samples were excited at $555 \mathrm{~nm}$ and $645 \mathrm{~nm}$, respectively. Data are plotted relative to the emission maxima of the D/AT34C/T110C CaM only samples (black) which was normalized to $100 \%$. All samples were in $40 \mathrm{mM}$ HEPES (pH 7.6) supplemented with $150 \mathrm{mM} \mathrm{NaCl}, 1 \mathrm{mM} \mathrm{CaCl}_{2}, 10 \%$ glycerol in the presence of $5 \mu \mathrm{M} \mathrm{H}_{4} \mathrm{~B}$. 

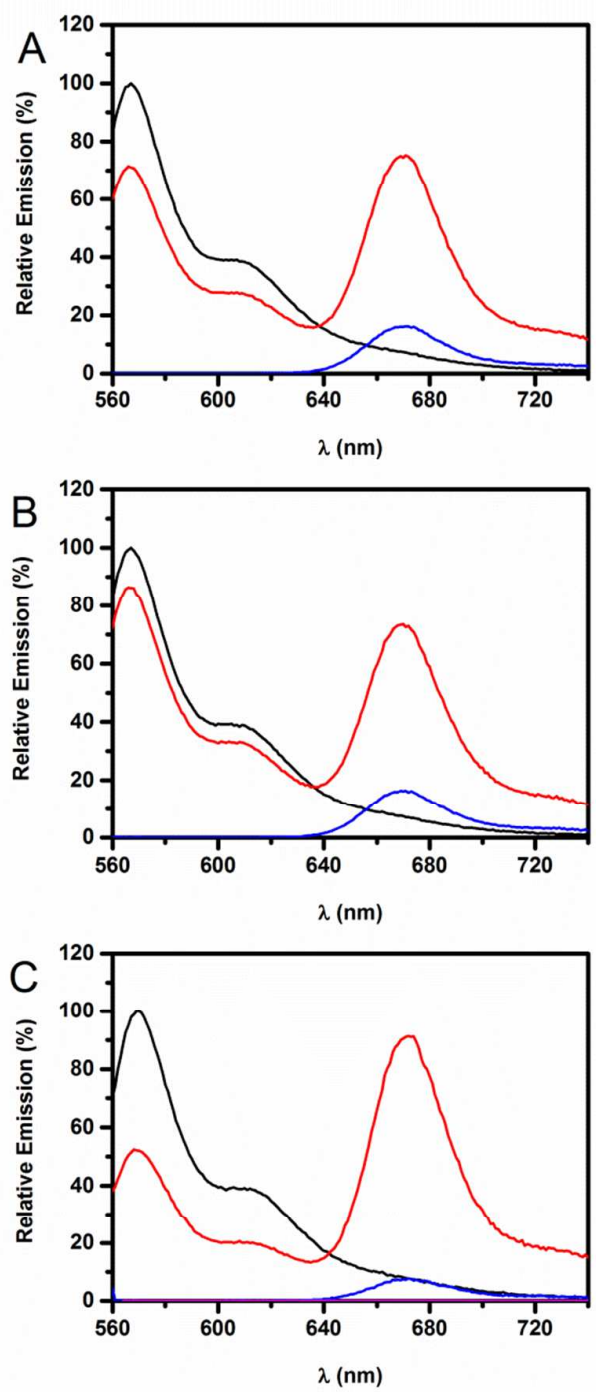

Figure S4. Fluorescence emission spectra of donor and acceptor fluorophores bound to T34C/T110C-CaM. A), B) and C) are representative of apo-CaM, $\mathrm{Ca}^{2+}$-bound $\mathrm{CaM}$ and $\mathrm{nNOS}$ with equimolar $\mathrm{Ca}^{2+}-$ bound $\mathrm{CaM}$, respectively. Black is $0.15 \mu \mathrm{M}$ donor only labelled sample (D-T34C/T110C-CaM), red is $0.3 \mu \mathrm{M}$ donor-acceptor labelled CaM (DA-T34C/T110C-CaM) and blue is $0.15 \mu \mathrm{M}$ acceptor labelled CaM (A-T34C/T110C-CaM). In C) the magenta line is $0.3 \mu \mathrm{M}$ nNOS bound to $0.3 \mu \mathrm{M}$ unlabelled T34C/T110C-CaM, excited at $555 \mathrm{~nm}$. All data are plotted relative to the emission maxima $(\sim 570 \mathrm{~nm}$ ) of the donor only sample (black). All data were recorded using a $555 \mathrm{~nm}$ excitation wavelength and all samples were in $40 \mathrm{mM} \mathrm{HEPES}$ (pH 7.6), $150 \mathrm{mM} \mathrm{NaCl,} 1 \mathrm{mM}$ $\mathrm{CaCl}_{2}, 10 \%$ glycerol in the presence of $5 \mu \mathrm{M} \mathrm{H}_{4} \mathrm{~B}$. 

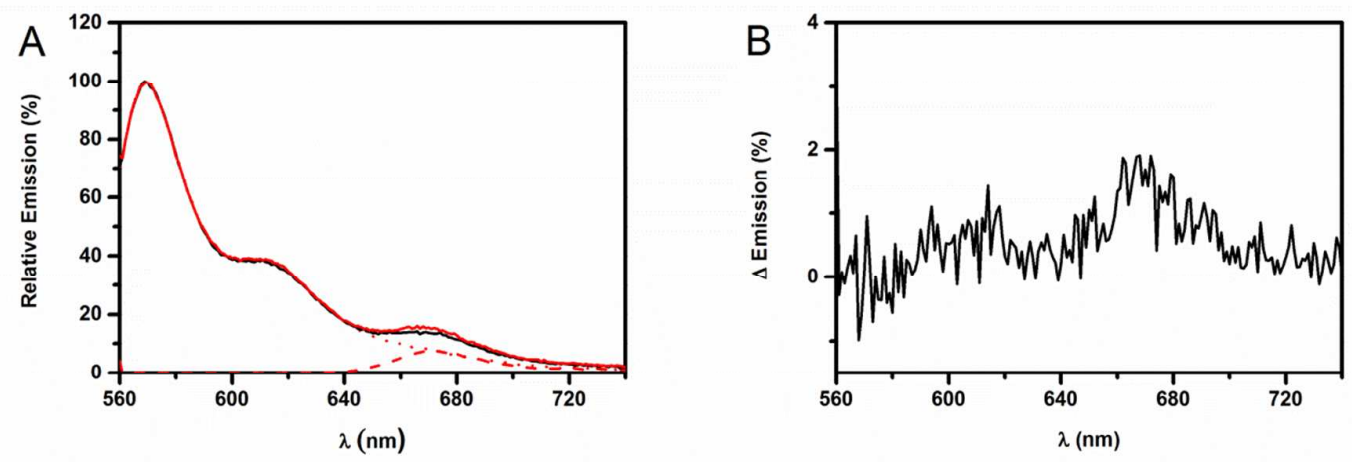

Figure S5. No Inter-CaM FRET across the nNOS dimer. The black spectrum in A) shows the fluorescence emission from $0.15 \mu \mathrm{M}$ of D-T34C/T110C CaM and $0.15 \mu \mathrm{M}$ A-T34C/T110C CaM bound to $0.3 \mu \mathrm{M}$ oxidized nNOS (black). The red spectrum in A) show the emission from $0.15 \mu \mathrm{M}$ single labelled D-T34C/T110C CaM bound to one equivalent of nNOS (dotted) as well as the emission spectrum of $0.15 \mu \mathrm{M} \mathrm{A-T34C/T110C} \mathrm{sample}$ bound to equimolar nNOS (dash red). The sum of the two aforementioned spectra in $A$ ) is shown as a solid red line. All data in A) are normalized to the concentration of donor fluorophore in the D-T34C/T110C CaM bound nNOS containing sample (dotted red line). B) shows the difference in relative fluorescence emission between the solid red and black spectra shown in A) (red spectrum - black spectrum) to demonstrate that with using the A555-A647 fluorophore pair $\left(R_{0}=47 \AA\right)$ there is no recordable FRET between the two calmodulins bound to the nNOS dimer. All data were recorded using a $555 \mathrm{~nm}$ excitation and all samples were in $40 \mathrm{mM}$ HEPES (pH 7.6), $150 \mathrm{mM} \mathrm{NaCl}, 1 \mathrm{mM} \mathrm{CaCl}_{2}, 10 \%$ glycerol in the presence of $5 \mu \mathrm{M} \mathrm{H}_{4} \mathrm{~B}$. 
2.3 Stopped-flow UV-Vis Measurements.
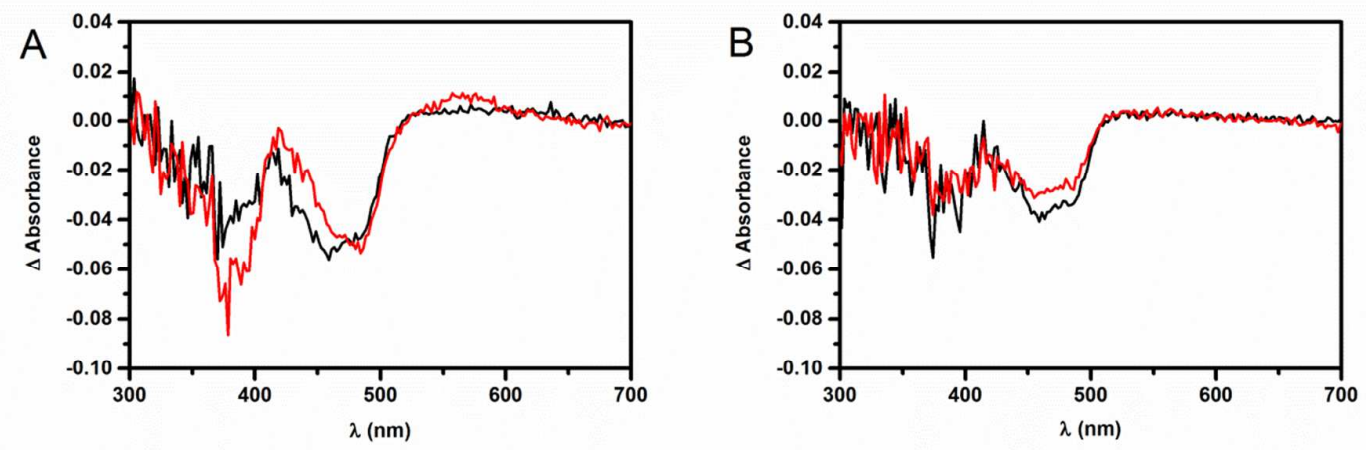

Figure S6. Absorbance changes, recorded by a photodiode array (PDA) stopped-flow instrument, $10 \mathrm{~s}$ after mixing of $5 \mu \mathrm{M}$ (final concentration) of A) native or B) 5 -dFMN-reconstituted NOS with 20-fold excess NADPH in the presence (red) and absence (black) of CaM. All experiments were conducted at $10{ }^{\circ} \mathrm{C}$ in $40 \mathrm{mM}$ HEPES (pH 7.6) supplemented with $150 \mathrm{mM} \mathrm{NaCl}, 1 \mathrm{mM} \mathrm{CaCl}, 10 \%$ glycerol with $1 \mathrm{mM} \mathrm{CaCl}_{2}$. 
Table S2 Observed KIE values for NADPH-driven nNOS flavin reduction

\begin{tabular}{|c|c|c|c|c|c|}
\hline NOS & NADPH & $k_{1}$ & $k_{2}$ & $k_{3}$ & $k_{4}$ \\
\hline Native & $\begin{array}{l}\text { Pro- } R \\
\text { NADP }^{2} \mathrm{H}\end{array}$ & $8.9(2.8)$ & $7.6(5)$ & $2.7(1.1)$ & $2.3(1.4)$ \\
\hline Native +CaM & $\begin{array}{l}\text { Pro- } R \\
\text { NADP }^{2} \mathrm{H}\end{array}$ & $4.3(0.6)$ & $2.4(4.6)$ & $6(8.5)$ & 1.7 (1.3) \\
\hline Native & $\begin{array}{l}\text { Pro-S } \\
\text { NADP }^{2} \mathrm{H}\end{array}$ & $1.1(0.6)$ & $1.1(1.1)$ & $1.4(0.6)$ & $1.8(1.0)$ \\
\hline Native +CaM & $\begin{array}{l}\text { Pro-S } \\
\text { NADP }^{2} \mathrm{H}\end{array}$ & $1.4(0.2)$ & $2.1(4.1)$ & $2.7(3.8)$ & $1.1(1.0)$ \\
\hline
\end{tabular}

Estimated errors are given in parenthesis. 


\subsection{Stopped-flow FRET measurements.}
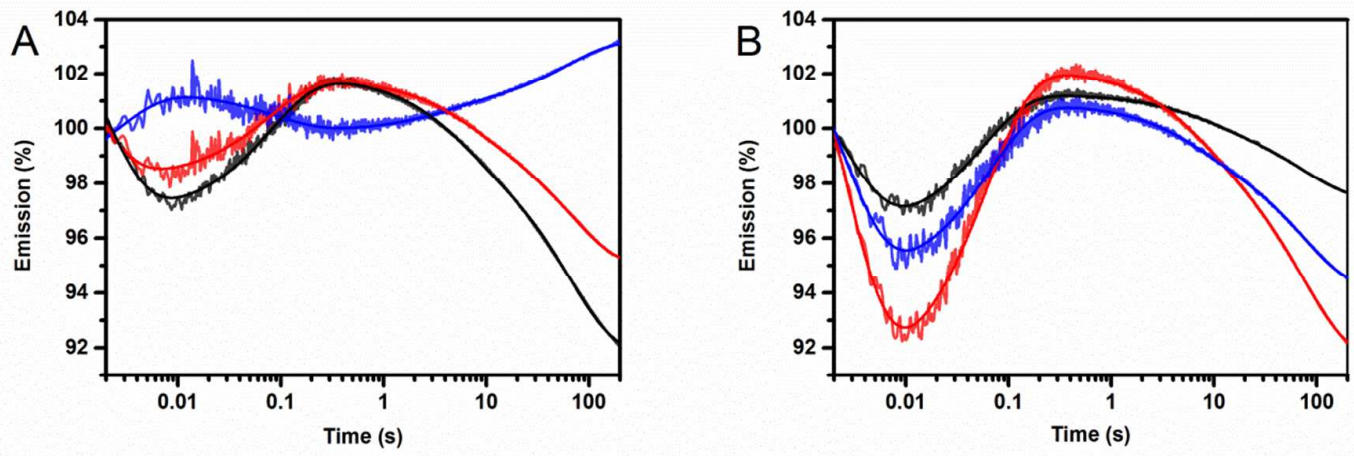

Figure S7. Time-dependent changes in fluorescence emission of fluorophores bound to the $0.3 \mu \mathrm{M}$ singlelabelled (donor or acceptor labelled - black) or $0.3 \mu \mathrm{M}$ double-labelled (donor and acceptor labelled - red) T34C/T110C CaM-oxidized nNOS complex upon reduction with excess NADPH (100 $\mu \mathrm{M}$, final concentration). No emission changes were recorded in the dead-time of the stopped-flow instrument and all data were normalized to percentage of emission change, relative to the emission at 0.002 seconds. The difference between the fluorophore emissions in the single- (donor or acceptor labelled T34C/T110C CaM) and doublelabelled samples (donor and acceptor labelled T34C/T110C CaM) were normalized to percentage emission change at time 0.002 seconds and is shown as the blue transient. Donor emission changes are monitored using a $600 \mathrm{~nm}(+/-10 \mathrm{~nm})$ band width pass (BWP) with a $555 \mathrm{~nm}$ excitation wavelength and are shown in panel A). Acceptor emission changes are followed using a $650 \mathrm{~nm}$ long-pass (cut-on) filter, data collected for the acceptor only single labelled sample (A-T34C/T110C CaM-nNOS) were collected using a $645 \mathrm{~nm}$ excitation wavelength while the emission changes for the acceptor in the double labelled sample were collected with a $555 \mathrm{~nm}$ excitation wavelength. All fluorescence changes associated with the acceptor are shown in panel B). All experiments were conducted in $40 \mathrm{mM} \mathrm{HEPES}$ (pH 7.6), $150 \mathrm{mM} \mathrm{NaCl}, 1 \mathrm{mM} \mathrm{CaCl} 2,10 \%$ glycerol with $5 \mu \mathrm{M}$ $\mathrm{H}_{4} \mathrm{~B}$. Transients shown here are averages of 8 traces. 

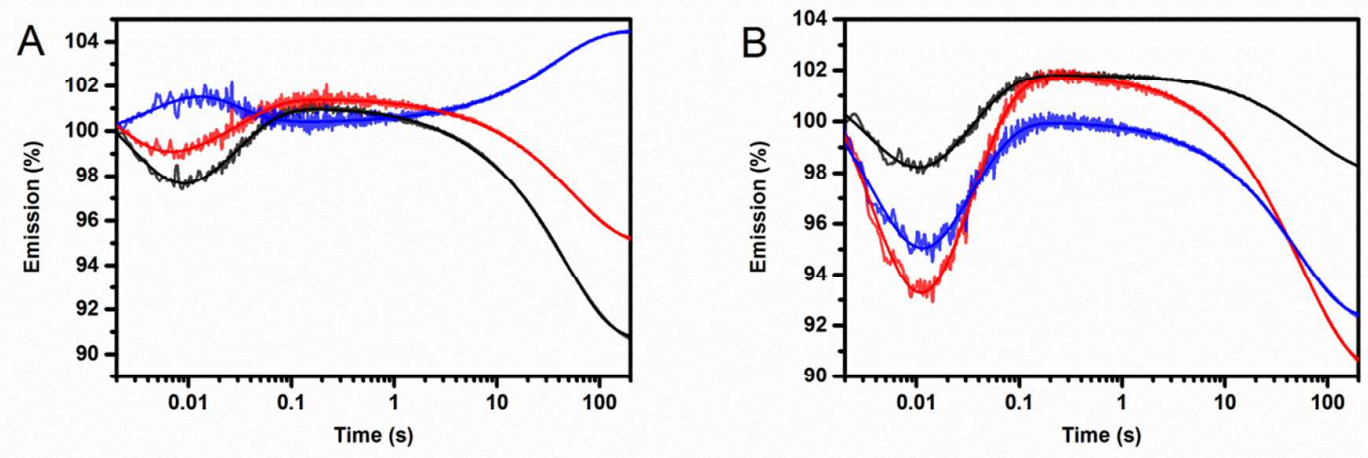

Figure S8. Time-dependent changes in fluorescence emission of fluorophores bound to the $0.3 \mu \mathrm{M}$ singlelabelled (donor or acceptor labelled - black) or $0.3 \mu \mathrm{M}$ double-labelled (donor and acceptor labelled - red) T34C/T110C CaM-oxidized 5-dFMN nNOS complex upon reduction with excess NADPH (100 $\mu$ M, final concentration). No emission changes were recorded in the dead-time of the stopped-flow instrument and all data were normalized to percentage of emission change, relative to the emission at 0.002 seconds. The difference between the fluorophore emissions in the single- (donor or acceptor labelled T34C/T110C CaM) and double-labelled samples (donor and acceptor labelled T34C/T110C CaM) were normalized to percentage emission change at time 0.002 seconds and is shown as the blue transient. Donor emission changes are monitored using a $600 \mathrm{~nm}(+/-10 \mathrm{~nm}$ ) band width pass (BWP) with a $555 \mathrm{~nm}$ excitation wavelength and are shown in panel A). Acceptor emission changes are followed using a $650 \mathrm{~nm}$ long-pass (cut-on) filter, data collected for the acceptor only single labelled sample (A-T34C/T110C CaM-5-dFMN nNOS) were collected using a $645 \mathrm{~nm}$ excitation wavelength while the emission changes for the acceptor in the double labelled sample were collected with a $555 \mathrm{~nm}$ excitation wavelength. All fluorescence changes associated with the acceptor are shown in panel B). All experiments were conducted in $40 \mathrm{mM} \mathrm{HEPES}$ (pH 7.6), $150 \mathrm{mM} \mathrm{NaCl}, 1 \mathrm{mM} \mathrm{CaCl}, 10 \%$ glycerol with $5 \mu \mathrm{M} \mathrm{H}_{4} \mathrm{~B}$. Transients show here are averages of 8 traces. 
Table S3. Donor and acceptor fluorophore emission changes extracted from fitting to transients seen in Figure 4A and 4C (de-convoluted FRET) and Figure S7 (native) and S8 (5-dFMN).

\begin{tabular}{|c|c|c|c|c|c|c|c|c|c|c|}
\hline NOS & & $k_{1}\left(\mathrm{~s}^{-1}\right)$ & $\Delta \mathrm{C}_{1}$ & $k_{2}\left(\mathrm{~s}^{-1}\right)$ & $\Delta C_{2}$ & $k_{3}\left(\mathrm{~s}^{-1}\right)$ & $\Delta \mathrm{C}_{3}$ & $k_{4}\left(\mathrm{~s}^{-1}\right)$ & $\Delta \mathrm{C}_{4}$ & $\mathrm{y} 0$ \\
\hline \multirow[t]{6}{*}{ native } & SL Donor & $504(86)$ & $9.8(3.7)$ & $11.7(1.3)$ & $-4.9(0.8)$ & $0.19(0.03)$ & $2.8(0.3)$ & $0.016(0.001)$ & $7.3(0.1)$ & $91.3(0.8)$ \\
\hline & DL Donor & $663(262)$ & $3.5(2.3)$ & $11.6(1.8)$ & $-3.5(0.7)$ & $0.19(0.01)$ & $1.7(0.1)$ & $0.014(0.001)$ & $4.9(0.1)$ & $95.4(0.9)$ \\
\hline & Donor (FRET) & $259(125)$ & $-2.9(0.7)$ & $14.2(5.2)$ & $1.8(1.0)$ & $0.25(0.16)$ & $-0.9(0.5)$ & $0.018(0.003)$ & $-2.5(0.2)$ & $103.6(1.3)$ \\
\hline & SL Acceptor & $438(116)$ & $9.5(2.8)$ & $17.2(1.2)$ & $-5.1(0.4)$ & $0.13(0.02)$ & $1.0(0.1)$ & $0.013(0.001)$ & $2.8(0.2)$ & $98.0(1.0)$ \\
\hline & DL Acceptor & $417(68)$ & $21.6(4.5)$ & $15.8(1.2)$ & $-11.5(1.2)$ & $0.15(0.01)$ & $2.8(0.3)$ & $0.013(0.001)$ & $7.7(0.2)$ & $92.1(1.2)$ \\
\hline & Acceptor (FRET) & $297(61)$ & $8.1(1.9)$ & $15.1(2.4)$ & $-6.4(1.2)$ & $0.17(0.02)$ & $1.7(0.2)$ & $0.014(0.002)$ & $5.0(0.3)$ & $94.3(2.3)$ \\
\hline \multirow[t]{6}{*}{ 5-dFMN } & SL Donor & $239(68)$ & $6.3(1.3)$ & $38.8(6.0)$ & $-5.9(2.0)$ & $0.22(0.1)$ & $1.1(0.2)$ & $0.021(0.001)$ & $9.2(0.2)$ & $90.3(1.2)$ \\
\hline & DL Donor & $295(116)$ & $3.4(1.1)$ & $37.2(12.2)$ & $-3.7(0.9)$ & $0.61(1.2)$ & $0.7(0.2)$ & $0.018(0.001)$ & $5.7(0.2)$ & $95.0(1.2)$ \\
\hline & Donor (FRET) & $240(79)$ & $-2.7(1.3)$ & $28.9(20.1)$ & $1.7(1.1)$ & $1.13(1.14)$ & $-0.4(0.1)$ & $0.030(0.004)$ & $-3.7(0.2)$ & $104.7(1.3)$ \\
\hline & SL Acceptor & 201 (39) & $5.7(1.0)$ & $31.6(4.2)$ & $-6.1(1.0)$ & $0.04(0.02)$ & $1.2(0.5)$ & $0.010(0.002)$ & $2.8(0.4)$ & $97.6(0.9)$ \\
\hline & DL Acceptor & $189(19)$ & $15.7(1.6)$ & $32.5(2.1)$ & $-14.9(1.6)$ & $0.09(0.04)$ & $1.8(0.6)$ & $0.016(0.001)$ & $9.8(0.6)$ & $90.6(2.2)$ \\
\hline & Acceptor (FRET) & $186(42)$ & $10.1(1.4)$ & $33.4(6.0)$ & $-8.8(1.2)$ & $0.18(0.08)$ & $0.9(0.3)$ & $0.019(0.002)$ & $7.0(0.4)$ & 93.0 (1.9) \\
\hline
\end{tabular}

Estimated errors are given in parenthesis. SL, single labelled; DL, double labelled. 

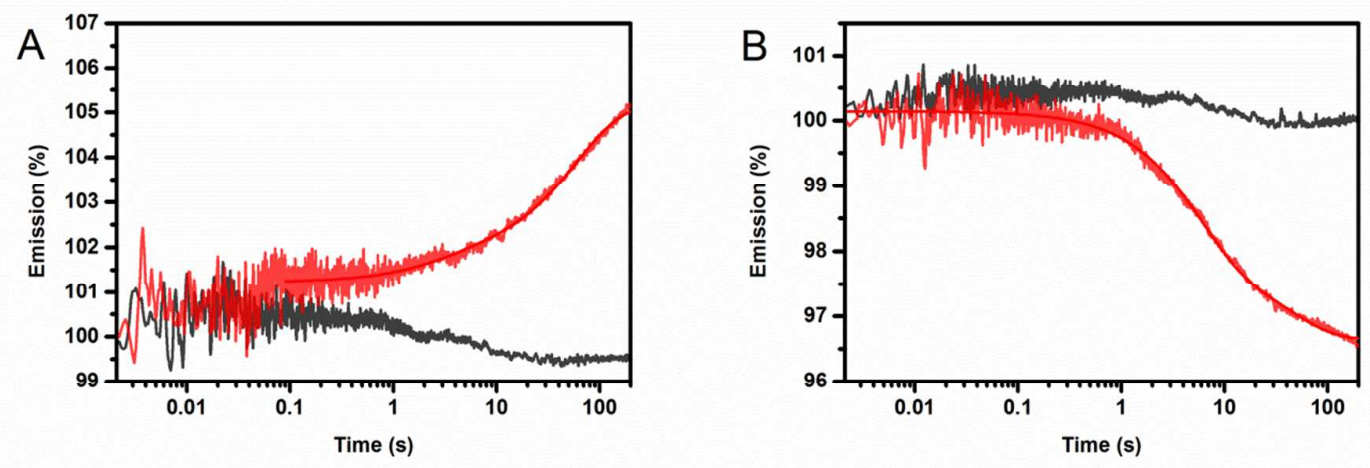

Figure S9. Time-dependent changes in fluorescence emission of fluorophores bound to the $0.3 \mu \mathrm{M}$ singlelabelled (donor or acceptor labelled - black) or $0.3 \mu \mathrm{M}$ double-labelled (donor and acceptor labelled - red) T34C/T110C CaM-oxidized nNOS complex upon reduction with excess NADP ${ }^{+}$(500 $\mu \mathrm{M}$, final concentration). No emission changes were recorded in the dead-time of the stopped-flow instrument and all data were normalized to percentage of emission change, relative to the emission at 0.002 seconds after mixing. The difference between the fluorophore emissions in the single- (donor or acceptor labelled T34C/T110C CaM) and double-labelled samples (donor and acceptor labelled T34C/T110C CaM) were not calculated since little changes were observed in the single labelled samples (black) which are attributed to photo-bleaching (see results and discussion). Donor emission changes are monitored using a $600 \mathrm{~nm}(+/-10 \mathrm{~nm})$ band width pass (BWP) with a $555 \mathrm{~nm}$ excitation wavelength and are shown in panel A). Acceptor emission changes shown in B) were followed using a $650 \mathrm{~nm}$ long-pass (cut-on) filter, data collected for the acceptor only single labelled sample (A-T34C/T110C CaM-nNOS) were collected using a $645 \mathrm{~nm}$ excitation wavelength while the emission changes for the acceptor in the double labelled sample were collected with a $555 \mathrm{~nm}$ excitation wavelength. All fluorescence changes associated with the acceptor are shown in panel B). All experiments were conducted in $40 \mathrm{mM}$ HEPES (pH 7.6), $150 \mathrm{mM} \mathrm{NaCl}, 1 \mathrm{mM} \mathrm{CaCl}_{2}, 10 \%$ glycerol with $5 \mu \mathrm{M} \mathrm{H}_{4} \mathrm{~B}$. Transients show here are averages of 8 traces. 
Table S4. Donor and acceptor fluorophore emission changes extracted from fitting exponential functions to transients seen in Figure 6 and Figure S9.

\begin{tabular}{llllll}
\hline & $k_{1}\left(\mathrm{~s}^{-1}\right)$ & $\Delta \mathrm{C}_{1}$ & $k_{2}\left(\mathrm{~s}^{-1}\right)$ & $\Delta \mathrm{C}_{2}$ & $\mathrm{y} 0$ \\
\hline SL D & $\mathrm{ND}$ & $\mathrm{ND}$ & $\mathrm{ND}$ & $\mathrm{ND}$ & $\mathrm{ND}$ \\
DL D & $1.24(1.2)$ & $-0.7(0.2)$ & $0.017(0.003)$ & $-3.9(0.7)$ & $105.0(1.1)$ \\
\hline Donor (FRET) & $1.24(1.2)$ & $-0.7(0.2)$ & $0.017(0.003)$ & $-3.9(0.7)$ & $105.0(1.1)$ \\
\hline SL A & $\mathrm{ND}$ & $\mathrm{ND}$ & $\mathrm{ND}$ & $\mathrm{ND}$ & $\mathrm{ND}$ \\
DL A & $0.53(0.97)$ & $1.7(1.8)$ & $0.055(0.057)$ & $1.8(1.1)$ & $97.8(2.5)$ \\
\hline Acceptor (FRET) & $0.53(0.97)$ & $1.7(1.8)$ & $0.055(0.057)$ & $1.8(1.1)$ & $97.8(2.5)$ \\
\hline \multirow{2}{*}{ Donor/Acceptor } & $0.43(0.56)$ & $(0.012)$ & $0.020(0.011)$ & $(0.013)$ & $1.10(0.02)$
\end{tabular}

Estimated errors are given in parenthesis. SL, single labelled; DL, double labelled. ND, not determined. 
2.5 Circular dichroism (CD) measurements.
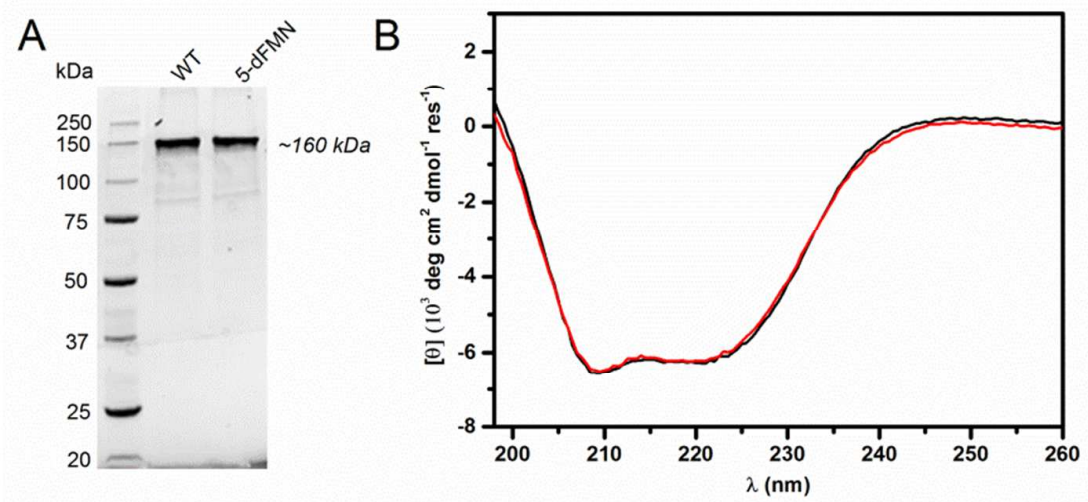

Figure S10. A) SDS-PAGE gel of native and 5-dFMN nNOS showing no alteration (proteolysis) in nNOS monomer after unfolding treatment with potassium bromide and subsequent protein refolding (see Experimental Section). B) Circular dichroism of native (black) and 5-dFMN nNOS (red) showing secondary structure is identical between the two enzymes. CD experiments were performed on $5 \mu \mathrm{M}$ of both native and 5-dFMN nNOS in 40 mM HEPES (pH 7.6) supplemented with $150 \mathrm{mM} \mathrm{NaCl}$ and $10 \%$ glycerol. 

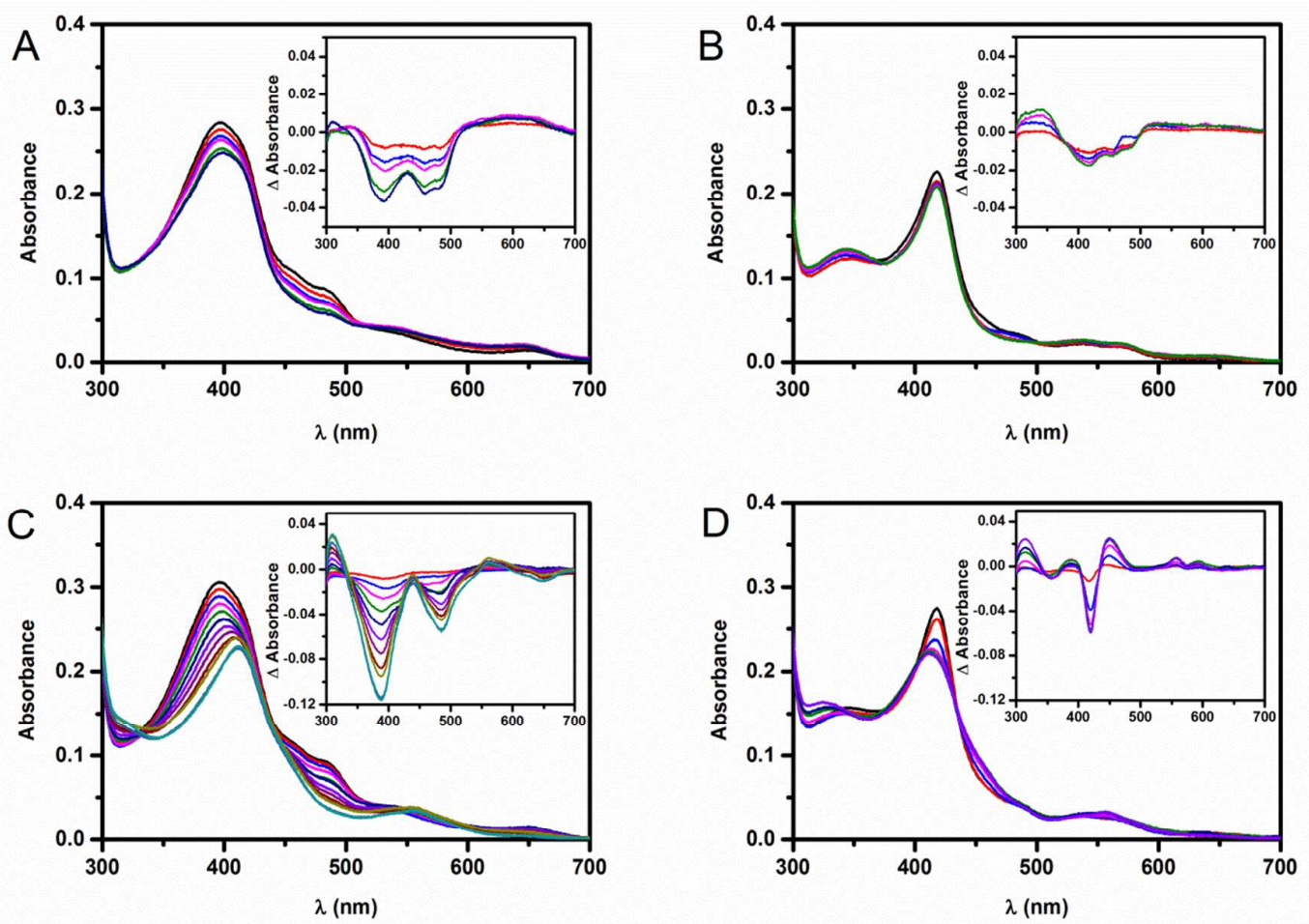

Figure S11. Reductive titration of native and 5-dFMN reconstituted NOS. NOS ( 3 $\mu \mathrm{M})$ was anaerobically titrated with NADPH ( $1 \mu \mathrm{M}$ aliquots) and dithionite (DT) in the presence of $5 \mathrm{mM}$ L-arginine and $5 \mu \mathrm{M} \mathrm{H}_{4} \mathrm{~B}$ in $40 \mathrm{mM}$ HEPES (pH 7.6) buffer supplemented with $10 \%$ glycerol and $150 \mathrm{mM} \mathrm{NaCl}$. Spectra were taken after each addition. A) Native NOS titrated with NADPH, B) 5-dFMN NOS titrated with NADPH, C) Native NOS titrated with dithionite, and D) 5-dFMN substituted NOS titrated with DT. The insets show the observed difference spectra upon addition of reductant. Spectra were recorded at ambient room temperature on a Cary UV-Vis spectrometer with $10-20 \mu \mathrm{M}$ of flavin samples. 
A

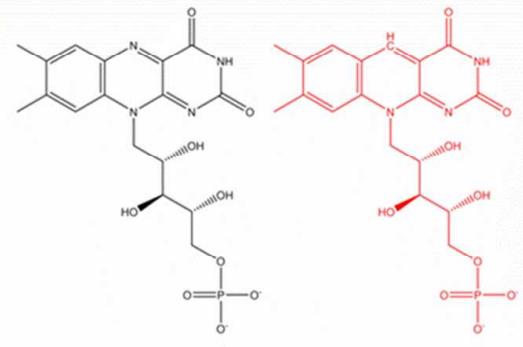

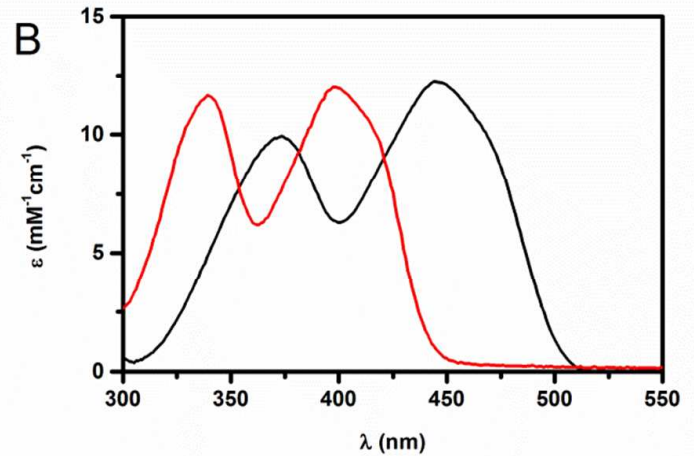

Figure S12. A) Structures of FMN (black) and 5-dFMN (red). B) The absorbance spectra of flavin mononucleotide (FMN; black) and 5-deazaflavin mononucleotide (5-dFMN; red) in $40 \mathrm{mM} \mathrm{HEPES} \mathrm{(pH} \mathrm{7.6)}$ supplemented with $150 \mathrm{mM} \mathrm{NaCl}$ and $10 \%$ glycerol. Spectra were recorded at ambient room temperature on a Cary UV-Vis spectrometer with $10-20 \mu \mathrm{M}$ of flavin samples. Spectra in B) are normalized to molar extinction coefficient. 


\section{References}

(1) Hay, S.; Pudney, C. R.; Scrutton, N. S. FEBS J. 2009, 276, 3930-3941.

(2) Nakagawa, S.; Igarashi, A.; Ohta, T.; Hagihara, T.; Fujio, T.; Aisaka, K. Biosci., Biotechnol., Biochem. 1995, 59, 694-702.

(3) lamurri, S. M.; Daugherty, A. B.; Edmondson, D. E.; Lutz, S. Protein Engineering Design \& Selection 2013, 26, 791-795.

(4) Sobolewska-Stawiarz, A.; Leferink, N. G. H.; Fisher, K.; Heyes, D. J.; Hay, S.; Rigby, S. E. J.; Scrutton, N. S. J. Biol. Chem. 2014, 289, 11725-11738.

(5) Stuehr, D. J. Methods Enzymol. 1996, 268, 324-333.

(6) Adak, S.; Aulak, K. S.; Stuehr, D. J. J. Biol. Chem. 2001, 276, 23246-23252.

(7) Wilson, M. A.; Brunger, A. T. J. Mol. Biol. 2000, 301, 1237-1256.

(8) Roman, L. J.; Martasek, P.; Miller, R. T.; Harris, D. E.; de la Garza, M. A.; Shea, T. M.; Kim, J. J. P.; Masters, B. S. S. J. Biol. Chem. 2000, 275, 29225-29232.

(9) Panda, K.; Ghosh, S.; Stuehr, D. J. J. Biol. Chem. 2001, 276, 23349-23356.

(10) Sagami, I.; Daff, S.; Shimizu, T. J. Biol. Chem. 2001, 276, 30036-30042.

(11) Knight, K.; Scrutton, N. S. Biochem. J. 2002, 367, 19-30. 